\title{
Modelling of the neutron production in a mixed beam DT neutron generator
}

pÿufar, Alja

Elsevier

2018

pÿufar , A , JET Contributors \& Ahlgren , T 2018, Modelling of the neutron production in a mixed beam DT neutron generator . in S Cho , M-Y Ahn , M Akiba, K Feng , A Ibarra , M Q Tran \& H Hashizume (eds), Proceedings of the 13th International Symposium on Fusion Nuclear Technology (ISFNT-13) . Fusion Engineering and Design , no. Part B , vol. 136 , Elsevier , Amsterdam , pp. 1089-1093, International Symposium on Fusion Nuclear Technology (ISFNT) , Kyoto , Japan , 25/09/2017 . https://doi.org/10.1016/j.fusengdes.2018.04.075

http://hdl.handle.net/10138/326702

https://doi.org/10.1016/j.fusengdes.2018.04.075

unspecified

submittedVersion

Downloaded from Helda, University of Helsinki institutional repository.

This is an electronic reprint of the original article.

This reprint may differ from the original in pagination and typographic detail.

Please cite the original version. 
WPJET3-CPR(17) 17303

A Cufar et al.

\section{Modelling of the Neutron Production in the Mixed Beam DT neutron generator}

Preprint of Paper to be submitted for publication in Proceeding of 13th International Symposium on Fusion Nuclear Technology (ISFNT)

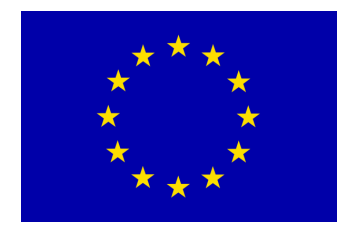

This work has been carried out within the framework of the EUROfusion Consortium and has received funding from the Euratom research and training programme 2014-2018 under grant agreement No 633053. The views and opinions expressed herein do not necessarily reflect those of the European Commission. 
This document is intended for publication in the open literature. It is made available on the clear understanding that it may not be further circulated and extracts or references may not be published prior to publication of the original when applicable, or without the consent of the Publications Officer, EUROfusion Programme Management Unit, Culham Science Centre, Abingdon, Oxon, OX14 3DB, UK or e-mail Publications.Officer@euro-fusion.org

Enquiries about Copyright and reproduction should be addressed to the Publications Officer, EUROfusion Programme Management Unit, Culham Science Centre, Abingdon, Oxon, OX14 3DB, UK or e-mail Publications.Officer@euro-fusion.org

The contents of this preprint and all other EUROfusion Preprints, Reports and Conference Papers are available to view online free at http://www.euro-fusionscipub.org. This site has full search facilities and e-mail alert options. In the JET specific papers the diagrams contained within the PDFs on this site are hyperlinked 


\title{
Modelling of the Neutron Production in a Mixed Beam DT Neutron Generator
}

\author{
Aljaž Čufar ${ }^{\mathrm{a}}$, Paola Batistoni ${ }^{\mathrm{b}}$, Zamir Ghani ${ }^{\mathrm{c}}$, Luca Giacomelli ${ }^{\mathrm{d}}$, Igor Lengar ${ }^{\mathrm{a}}$, Stefano Loreti ${ }^{\mathrm{b}}$, \\ Alberto Milocco ${ }^{\mathrm{e}}$, Sergey Popovichev ${ }^{\mathrm{c}}$, Mario Pillon ${ }^{\mathrm{b}}$, Davide Rigamonti ${ }^{\mathrm{d}, \mathrm{e}}$, Marica Rebai ${ }^{\mathrm{d}, \mathrm{e}}$, \\ Marco Tardocchi ${ }^{\mathrm{d}}$, Luka Snoj ${ }^{\mathrm{a}, \mathrm{f}}$, JET contributors ${ }^{1}$ \\ EUROfusion consortium, Culham Science Centre, Abington, United Kingdom \\ ${ }^{a}$ Jožef Stefan Institute, Ljubljana, Slovenia \\ ${ }^{b}$ ENEA, I-00044 Frascati, Rome, Italy \\ ${ }^{\circ} C C F E$, Abingdon, Oxon, OX14 3DB, United Kingdom \\ ${ }^{d}$ Istituto di Fisica del Plasma "P. Caldirola", CNR, Milano, Italy \\ eDipartimento di Fisica “G. Occhialini”, Università degli Studi di Milano-Bicocca, Milano, Italy \\ ${ }^{{ }^{f} F a c u l t y}$ of mathematics and physics, University of Ljubljana, Slovenia
}

\begin{abstract}
Compact DT neutron generators based on accelerators are often built on the principle of a mixed beam operation, meaning that deuterium (D) and tritium (T) are both present in the ion beam and in the target. Moreover, the beam consists of a mixture of ions and ionized molecules (D, T ions, and ionized D-D, T-T and D-T molecules) so the relevant source components come from $\mathrm{T}(\mathrm{d}, \mathrm{n}), \mathrm{D}(\mathrm{t}, \mathrm{n}), \mathrm{D}(\mathrm{d}, \mathrm{n})$ and $\mathrm{T}(\mathrm{t}, 2 \mathrm{n})$ reactions at different ion energies.

The method for assessing the relative amplitudes of different source components (DD, DT, TT) is presented. The assessment relies on the measurement of the neutron spectrum of different DT components $(\mathrm{T}(\mathrm{d}, \mathrm{n})$ and $\mathrm{D}(\mathrm{t}, \mathrm{n})$ at different energies) using a high resolution neutron spectrometer, e.g. a diamond detector, fusion reaction crosssections, and simulations of neutron generation in the target. Through this process a complete description of the neutron source properties of the mixed beam neutron generator can be made and a neutron source description card, in a format suitable for Monte Carlo code MCNP, produced.
\end{abstract}

Keywords: DT generator, mixed beam, neutron emission, ENEA-JSI subroutine, MCUNED

author's email: aljaz.cufar@ijs.si 


\section{Introduction}

Compact DT neutron generators suitable for use in calibrations of neutron yield detectors to DT neutrons can be based on mixed beam operation in which mixed beam and target, i.e. D and $\mathrm{T}$ are present in both target and the beam, are used to prolong the operational life of the generator by continuously implanting $\mathrm{D}$ and $\mathrm{T}$ in the target during the generator's operation. Due to the lack of an analyzing magnet, the ion beam consists of both ions and ionized molecules.

The nuclei interacting in the target can thus originate from ions $\left(\mathrm{D}^{+}\right.$, $\mathrm{T}^{+}$) or ionized molecules $\left(\mathrm{D}-\mathrm{D}^{+}, \quad \mathrm{D}-\mathrm{T}^{+}, \quad \mathrm{T}-\mathrm{T}^{+}\right)$ accelerated to energy defined by the acceleration system. Heavier (triatomic) molecules in the beam are neglected. The nuclei originating from ions receive full acceleration energy $\left(E_{\text {ion }}\right)$ while the nuclei from ionized molecules only receive a part of the energy based on their mass relative to the total mass of the molecule. This results in complex neutron emission properties with six distinct DT, three DD, and three TT source components. However, due to low relative intensity of DD and TT source components, only DD and TT neutrons generated by $\mathrm{D}^{+}$and $\mathrm{T}^{+}$ beam ions can be considered in the source definition. In this case, the source description consists of eight components, i.e. six DT, one $\mathrm{DD}$, and one TT source component.
The results and experience presented in the present paper are based on the process used in the characterization of the ING-17 $14 \mathrm{MeV}$ neutron generator, provided by VNIIA (Russia), which was used in the latest calibration of neutron yield monitors at JET (intensity $\approx 2 \times 10^{8} \mathrm{n} /$ s) [1]. According to the supplier, both the generator beam and target contain about 50\% $\mathrm{D}$ and $50 \% \mathrm{~T}$.

\section{Relative intensities of different neutron source components}

\subsection{Fusion reaction cross-sections}

The cross-sections for fusion reactions of interest are in Fig. 1. At energies relevant for typical compact neutron generators (up to $110 \mathrm{keV}$ [2]), the crosssections for DD and TT reactions are significantly lower than for DT reaction. This limits the importance of the DD and TT components in typical generators with approximately equal split between $\mathrm{D}$ and $\mathrm{T}$ in both the target and ion beam [2].

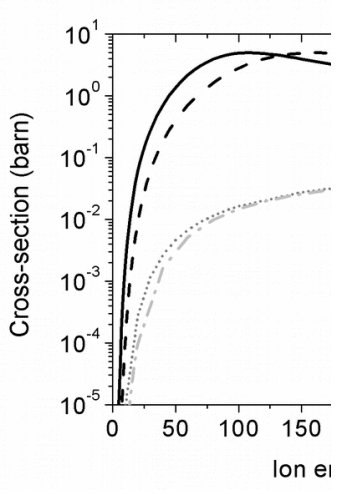

Fig. 1. Cross-sections for DT, DD and TT fusion reactions in the laboratory coordinate system [3].

\subsection{DT components}

In the mixed beam neutron generator based on the accelerator which accelerates positive ions and ionized molecules to energy $E_{\text {ion, }}$ in total six DT source components are expected. These components originate from fusion reactions:

- $\quad \mathrm{T}(\mathrm{d}, \mathrm{n})$ at deuterium ion energies $E_{\text {ion }}, 1 / 2$ $\mathrm{E}_{\text {ion }}$ and $2 / 5 \mathrm{E}_{\text {ion }}$, from $\mathrm{D}^{+}, \mathrm{D}-\mathrm{D}^{+}$and ${\mathrm{D}-\mathrm{T}^{+},}$respectively, and

- $\quad \mathrm{D}(\mathrm{t}, \mathrm{n})$ at tritium ion energies $E_{\text {ion }}, 1 / 2 E_{\text {ion }}$ and $3 / 5 \mathrm{E}_{\text {ion }}$ from $\mathrm{T}^{+}$, $\mathrm{T}_{-} \mathrm{T}^{+}$and $\mathrm{D}-\mathrm{T}^{+}$, respectively.

The spectra of each of the source components were calculated using the ENEA-JSI source subroutine [4] added to the standard MCNP6 [5]; however, other codes were also considered [6].

To perform the
neutron simulations, first the ion acceleration voltage needs to be known. This voltage does not necessarily correspond to the voltage indicated by the neutron generator control unit as there can be a significant drop in voltage between the high voltage generator and the ion acceleration stage of the neutron generator. However, as the acceleration voltage determines the energies of ions in $\mathrm{T}(\mathrm{d}, \mathrm{n})$ and $\mathrm{D}(\mathrm{t}$, n) fusion reactions, its determination is crucial for accurate simulation of the source components.

Once $\mathrm{E}_{\text {ion }}$ is determined, all six DT source components are simulated separately to get neutron emission spectra. Moreover, it can be assumed that the source components from D- $\mathrm{T}^{+}$molecules $(\mathrm{T}(\mathrm{d}, \mathrm{n})$ at $2 / 5$ and $\mathrm{D}(\mathrm{t}, \mathrm{n})$ at $3 / 5$ of $\left.E_{\text {ion }}\right)$ are $100 \%$ correlated. As suggested by the supplier and the literature [2], equal amounts of $\mathrm{D}$ and $\mathrm{T}$ in the target $(50 \% \mathrm{D}, 50 \% \mathrm{~T})$ were assumed. The neutron yields of different DT source components are shown in Fig. 2 and are given in Error: Reference source not found relative to the $\mathrm{T}(\mathrm{d}, \mathrm{n})$ component resulting from $\mathrm{D}^{+}$ions at $73 \mathrm{keV}$. Relative neutron yields and $100 \%$ correlation of $\mathrm{T}(\mathrm{d}, \mathrm{n})$ at $2 / 5 \mathrm{E}_{\text {ion }}$ and $\mathrm{D}(\mathrm{t}, \mathrm{n})$ at $3 / 5$ $\mathrm{E}_{\text {ion }}$ are then be used to decrease the number of fitting parameters to five.

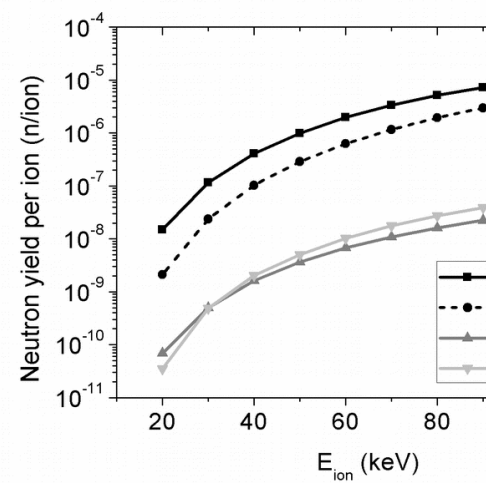

Fig. 2. Neutron yields, i.e. probabilities for neutron production per ion, of relevant reactions as a function of $E_{\text {ion }}$.

Table 1. Neutron yields of source components, i.e. probabilities for neutron production per ion, relative to the $\mathrm{T}(\mathrm{d}, \mathrm{n})$ component resulting from $\mathrm{D}^{+}$ions at $73 \mathrm{keV}$

\begin{tabular}{lll}
\hline $\begin{array}{l}\text { Fusion } \\
\text { reaction }\end{array}$ & $\begin{array}{l}\text { Ion energy } \\
(\mathrm{keV})\end{array}$ & $\begin{array}{l}\text { Rela } \\
\text { yield }\end{array}$ \\
\hline $\mathrm{T}(\mathrm{d}, \mathrm{n})$ & 73.0 & 100 \\
$\mathrm{~T}(\mathrm{~d}, \mathrm{n})$ & 36.5 & 7.24 \\
$\mathrm{~T}(\mathrm{~d}, \mathrm{n})$ & 29.2 & 2.66
\end{tabular}




\begin{tabular}{ll}
$\mathrm{D}(\mathrm{t}, \mathrm{n})$ & 73.0 \\
$\mathrm{D}(\mathrm{t}, \mathrm{n})$ & 36.5 \\
$\mathrm{D}(\mathrm{t}, \mathrm{n})$ & 43.8 \\
\hline
\end{tabular}

$$
\text { In }
$$

the

characterization of the neutron generator used for JET neutron monitor calibration, the ion acceleration voltage of $73 \mathrm{keV}$ was obtained by comparing the calculated neutron spectrum with the spectrum obtained by a high resolution spectroscopic

measurement using a diamond detector located directly in front of the neutron generator (Error: Reference source not found) [7], [8]. To perform the fitting, the detector's response function was used. This detector position is used due to the largest differences in energies of the peaks being at angles of $0^{\circ}$ and $180^{\circ}$ relative to the direction of the ion beam. The measured neutron spectrum could be reproduced by a linear combination of the six DT components described above. Generally the highest energy neutrons correspond to $\mathrm{D}(\mathrm{t}, \mathrm{n})$ at $\mathrm{T}^{+}$ion energies $\mathrm{E}_{\text {ion. }}$. More details on the fitting process can be found in [7], and results relevant for the compact DT neutron generator used in the latest calibration of the neutron yield detectors in JET [1] in Fig. 4 and Table 2.

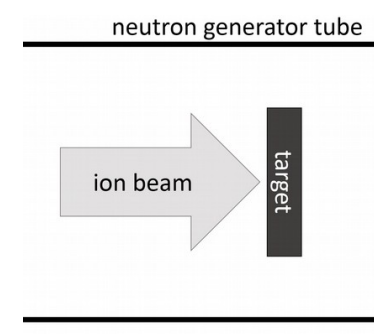

Fig. 3. Sketch of the neutron spectrum measurement process.

\section{DD and TT neutron} source components cannot be determined the same way as DT components due to the complex response function of the diamond detector [8] at energies relevant for DD leading to significantly decreased energy resolution, and due to wide emission spectrum of the TT component making it difficult to discern from the slowed down DT neutrons.

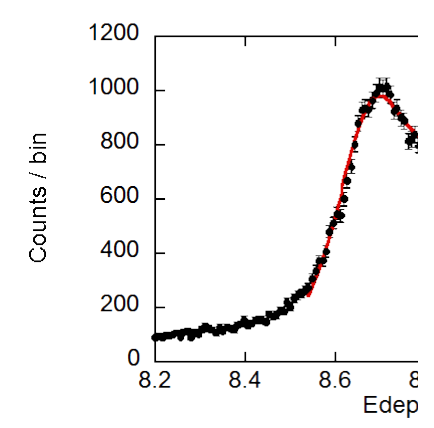

Fig. 4. Measured spectrum of $\alpha$ particles resulting from $\mathrm{C}(\mathrm{n}, \alpha)$ in diamond, and best fit of components from simulations [7].

Table 2. Relative intensities of DT neutron source components.

\begin{tabular}{ll}
$\begin{array}{l}\text { Fusion } \\
\text { reaction }\end{array}$ & $\begin{array}{l}\text { Ion energy } \\
(\mathrm{keV})\end{array}$ \\
\hline $\mathrm{T}(\mathrm{d}, \mathrm{n})$ & 73.0 \\
$\mathrm{~T}(\mathrm{~d}, \mathrm{n})$ & 36.5 \\
$\mathrm{~T}(\mathrm{~d}, \mathrm{n})$ & 29.2 \\
$\mathrm{D}(\mathrm{t}, \mathrm{n})$ & 73.0 \\
$\mathrm{D}(\mathrm{t}, \mathrm{n})$ & 36.5 \\
$\mathrm{D}(\mathrm{t}, \mathrm{n})$ & 43.8 \\
\hline
\end{tabular}

\subsection{Beam composition}

The measured neutron spectrum could be reproduced by a linear combination of the DT components described in the previous section. Using this linear combination, the ion beam components can be determined by the use of the relative neutron yields from neutron source simulations using the MCUNED code [10] (Error: Reference source not found) and the source component intensities from . For the target composition, typically equal concentrations of $\mathrm{D}$ and $T$, and the ratio of hydrogen to titanium atoms of up to 2 to 1 has been assumed [2]. The ion beam composition determined through this process is presented in Table 3.

Table 3. Ion beam composition determined from fitted neutron source component intensities and assumed target composition.

\begin{tabular}{ll}
\hline Ion beam & Rel \\
component & ion \\
\hline $\mathrm{D}^{+}$ & \\
$\mathrm{T}^{+}$ & \\
$\mathrm{D}-\mathrm{D}^{+}$ & \\
$\mathrm{T}-\mathrm{T}^{+}$ & \\
$\mathrm{D}-\mathrm{T}^{+}$ & \\
\hline
\end{tabular}

In principle the neutron spectrum can change throughout the operational life of the generator due to changes in its target and beam composition, e.g. as a result of $\mathrm{T}$ decay, and $\mathrm{D}$ or $\mathrm{T}$ building-up or escaping from the target. However, the neutron spectrum measurements at different times in the characterization process, i.e. spectra after different operational histories of the generator, showed no major changes in the neutron emission spectrum of the generator used.

\subsection{Relative importance of DD and TT components}

A first approximation for the upper limit of the relative intensities of the DD and TT source components is based entirely on the fusion reaction cross-sections. For both ion beam and target containing equal amounts of $\mathrm{D}$ and $\mathrm{T}$, the DD and TT contribution to the total neutron production can be calculated using Equations 1 and 2 . Contributions calculated based on the fusion cross-sections from Fig. 1 are in Fig. 5. $\sigma_{\mathrm{DT}}, \sigma_{\mathrm{TD}}$, $\sigma_{\mathrm{DD}}$, and $\sigma_{\mathrm{TD}}$ are the cross-sections for $\mathrm{T}(\mathrm{d}, \mathrm{n})$, $\mathrm{D}(\mathrm{t}, \mathrm{n}), \mathrm{D}(\mathrm{d}, \mathrm{n})$, and $\mathrm{T}(\mathrm{t}$, $2 \mathrm{n})$, respectively.

elative abundance in the

$$
D D \text { contribution }=\frac{\sigma_{D}}{\sigma_{D T}+\sigma_{T D}+c}
$$

$$
T T \text { contribution }=\frac{2 \cdot \sigma_{2}}{\sigma_{D T}+\sigma_{T D}+\sigma}
$$

The average energy at which the ion from the beam interacts with $\mathrm{D}$ or $\mathrm{T}$ in the target is lower than the acceleration energy $E_{\text {ion, }}$ hence the maximum of the relative DD and TT contributions calculated on the energy interval $\left[\mathrm{E}_{\min }, \mathrm{E}_{\text {ion }}\right]$ represent the upper limit of intensity of these components. $\mathrm{E}_{\min }$, the lowest energy that can realistically still give significant contribution to the neutron production, is selected depending on the $\mathrm{E}_{\text {ion }}$ and the cross-sections for fusion reactions (Fig. 1). For most cases $E_{\text {min }}$ between 10 and $20 \mathrm{keV}$ 
seems like a reasonable choice.

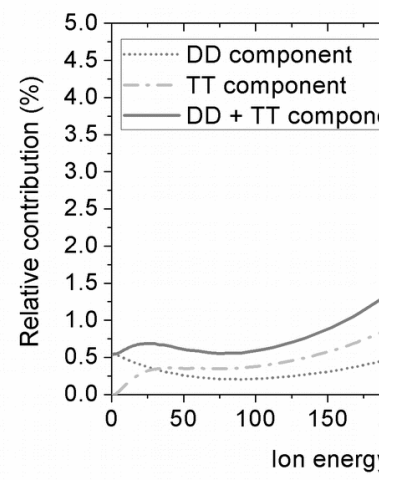

Fig. $5 . \quad$ Relative contributions of DD and TT source components to the total fusion cross-section as a function of ion energy.

For typical $\mathrm{E}_{\text {ion }}$ of up to $110 \mathrm{keV}$, the highest upper limit for the DD and TT source components seem to be approximately $\quad 0.4 \%$, while the combined intensity, i.e. the upper limit on the intensity of non DT source components, does not exceed $0.7 \%$ of the total neutron emission in Fig. 5. This means that in case of a compact neutron generator with typical $\mathrm{E}_{\text {ion }}$ (up to $110 \mathrm{keV}$ ) the DD and TT source components can in most cases be neglected unless $1 \%$ uncertainty represents significant addition to the total uncertainty. However, an increase of the acceleration voltage to $300 \mathrm{kV}$, as is typically done in neutron generators with higher source intensities, can potentially results in significantly higher intensities of components other than DT. In such cases the modelling of DD and TT components is advised. A possible use for the mixed beam neutron generator with relatively high intensity, and thus possibly high acceleration voltage, will be the calibration source used in calibration of neutron detectors to DT neutrons in ITER.

Finally, in order to assess the relative importance of DD and TT components, full simulation of the neutron source was performed in a simple model where only a thin $2 \mu \mathrm{m}$-target and $73 \mathrm{keV}$ ion beam were present. The simulation was repeated also for the case of a $10 \mu \mathrm{m}$ target and $300 \mathrm{keV}$ ion beam to compare with the case of a neutron generator with higher intensity. Such a model was chosen to minimize neutron scattering and thus to make it easier to separate different source components in the neutron emission spectrum. In our analysis the target consisted entirely of Ti-D-T, i.e. there were two hydrogen atoms per $\mathrm{Ti}$ atom, and an equal split between D and T was assumed. Each ion beam component, i.e. $\mathrm{D}^{+}$at $\mathrm{E}_{\text {ion }}, 1 / 2 \mathrm{E}_{\text {ion }}$ and 2/5 $\mathrm{E}_{\text {ion, }}$ and $\mathrm{T}^{+}$at $\mathrm{E}_{\mathrm{ion}}, 1 / 2$ $\mathrm{E}_{\text {ion }}$ and $3 / 5 \mathrm{E}_{\text {ion, }}$ can be simulated separately.

Due to relatively low amount of neutron scattering in the target, the different components in the emission spectra can be separated. There can be some difficulties in discerning between TT neutrons and downscattered DT neutrons; however, previous analyses of DT source with no DD or TT components showed that in geometry where only the thin target is filled with material, there is only $0.01 \%$ of neutrons at energies below the DT peak [11]. In the present analysis the downscattered DT neutrons were approximated as a constant background to DD and TT components. A more detailed and rigorous analysis was not deemed necessary due to the low number of neutrons scattered to lower energies.

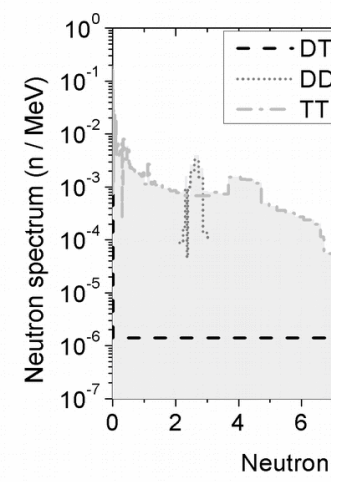

Fig. 6. The simulated neutron emission spectrum of a mixed beam neutron generator operating at 73 $\mathrm{keV}$ neutron generator. The colored area represents the sum of all components.

Results of the analysis are shown in Fig. 6 and Table 4. The analysis for the neutron generator with the same beam composition (ion beam composition from Table 3), i.e. approximation using the same kind of ion source, and acceleration voltage of $300 \mathrm{keV}$ was performed to test the performance at higher ion energies.

Based on these results, the DT neutron source components of a compact neutron generator used in JET's latest DT calibration represent roughly $99 \%$ of the neutrons emitted from its target. Even lower importance of the components other than DT were found for the neutron generator built on the same principle but with $300 \mathrm{kV}$ acceleration voltage. This is consistent with the fact that most of the ion beam represent $\mathrm{D}-\mathrm{T}^{+}$molecules (Table 3), slowing down of ions in the target and relative contributions shown in Fig. 5.

To take DD and TT components into account the relative intensities of different DT components need to be multiplied by the relative intensity of all DT components (roughly $99 \%$ in both presented cases), and the other two components added according to their relative intensities (Table 4).

Table 4. The relative intensity of the different neutron source components for $73 \mathrm{keV}$ and $300 \mathbf{~ k e V}$

acceleration energy.

\begin{tabular}{ll}
\hline Source component & Relative int \\
\hline $\mathrm{E}_{\text {ion }}$ & $73 \mathrm{keV}$ \\
\hline DD & 0.17 \\
TT & 0.96 \\
DT & 98.86 \\
\hline
\end{tabular}

\section{Preparation of source definition card for use in simulations}

\subsection{Properties of source components}

To quantify the details of different source components simulated using the ENEA-JSI source subroutine, each of the components was simulated separately. For each component $2 \times 10^{8}$ source events were recorded through MCNP's PTRAC functionality and analyzed using a Python script. In PTRAC file MCNP outputs all relevant information about the particle history, e.g. its position, direction, energy, and statistical weight. 
Additionally, filters can be applied to reduce the size of the file. In our case only the neutron source events were written in the PTRAC file. The Python script (Python script 1 in Fig. 7) sorted these source events according to their emission angle relative to the ion beam, and into energy bins within each angular interval. 400 angular bins (equidistant in cosine scale) and $10 \mathrm{keV}$ wide energy bins in relevant region were used to produce a high resolution description of the neutron source. The results of this process performed for each of the source components were 1D arrays describing the probabilities for emission in angular intervals, and 2D arrays describing neutron emission spectra for all emission angles.

When extracting the data, care needs to be taken that the energy and cosine boundaries do not cause rounding errors. As results in PTRAC file are shown in five digits, the cosines should be divided in intervals corresponding to the integer multiple of $10^{-6}$, and energies in integer multiple of $1 \mathrm{keV}$ for neutron energies above $10 \mathrm{MeV}$.

\subsection{Source definition card preparation}

Once the relative intensities of different source components are determined the source description (e.g. SDEF card in MCNP) can be produced. The definition consists of the probability of emission into each of the angular bins, and of relative probabilities for emission at different energies, which correspond to $1 \mathrm{D}$ and 2D arrays from previous step, respectively.

To construct a single source description the 1D and $2 \mathrm{D}$ arrays describing different source components are multiplied with their respective relative intensity (Table 2 for DT components, and importance of DD and TT components in Table 4) and summed into combined 1D and 2D arrays (Python script 2 in Fig. 7). Once the source is described with a single pair of $1 \mathrm{D}$ and $2 \mathrm{D}$ arrays it is output into a source description file (SDEF). The process is visually described in Fig. 7.

A single SDEF source description can be used to describe all source components. However, if the objective is to determine the separate effects of different source components it is advisable to prepare separate source definitions for each of the components or at least for each of the reactions, i.e. DT, DD and TT separately.

do for all source components

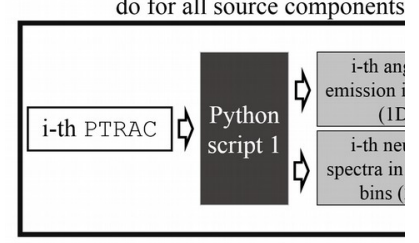

Fig. 7. Schematic description of data extraction and MCNP source definition card (SDEF) preparation.

Due to low relative contributions of the DD and TT neutron source components they can be for most uses in compact neutron generator applications either neglected or simplified using isotropic spectra, i.e. their angular dependence neglected. This can be justified by previous analyses which show that the majority of the anisotropy is caused by the generator's geometry and not by the anisotropy of fusion reactions [11].

\section{Conclusions}

The process of the neutron source spectrum analysis for a mixed beam DT neutron generator was proposed and demonstrated on a relevant example. Results show that in typical compact neutron generators with $\mathrm{E}_{\text {ion }}$ of up to $110 \mathrm{keV}$ the DD and TT neutron source components contribute less than $1 \%$ to the total neutron emission. Our analysis found that this is still the case, for typical beam and target composition, even when the acceleration voltage is increased to $300 \mathrm{kV}$, as is typical for neutron generators with higher intensities. However, given the ratios of crosssections, it is possible that contribution of components other than DT result in higher intensities under significantly different beam and target configurations. An example of relevant application for our analysis of source components is the use of an intense neutron generator for calibration of neutron detectors in future large fusion machines, e.g. ITER and DEMO, where DD and TT components will have to be taken into account if uncertainty in the neutron source will have to be significantly lower than 5\%. Additionally, the preparation process of the source definition card for use in simulations was explained.

\section{Acknowledgments}

This work has been carried out within the framework of the EUROfusion Consortium and has received funding from the Euratom research and training programme 2014-2018 under grant agreement No 633053. The views and opinions expressed herein do not necessarily reflect those of the European Commission.

The authors acknowledge the projects Development of methodology for calibration of neutron detectors with a 14.1 $\mathrm{MeV}$ neutron generator $\mathrm{J} 2-6752$, and Training of young researchers 1000-13-0106 were financially supported by the Slovenian Research Agency.

\section{References}

[1] P. Batistoni, et al., "Technical preparations for the in-vessel $14 \mathrm{MeV}$ neutron calibration at JET," Fus. Eng. Des., 117 , pp. 107-117 (2017).

[2] V. Valjković, "14 MeV Neutrons: Physics and Applications", CRC Press, Boca Raton, 2016.

[3] M.B. Chadwick, et al., "ENDF/B-VII.1:

Nuclear Data for Science and Technology: Cross 
Sections, Covariances, Fission Product Yields and Decay Data," Nuclear Data Sheets, 112, (2011).

[4] A. Milocco, et al., "A Monte Carlo model for low energy D-D neutron generators," NIMB, 271, pp. 6-12 (2012).

[5] T. Goorley, et al., "Initial MCNP6 Release Overview MCNP6 version 1.0," LA-UR-13-22934

(2013).

[6] A. Čufar, et al., "Comparison of DT neutron production codes MCUNED, ENEA-JSI source subroutine and DDT," Fus. Eng. Des, 109111, pp. 164-168 (2016).

[7] D. Rigamonti, et al., "Unprecedented very high resolution neutron spectroscopy measurements of 14 $\mathrm{MeV}$ neutrons and new prospects for diagnosing DT fusion plasmas," will be submitted to New Journal of Physics, 2017.

[8] P. Batistoni, et al., 14 $\mathrm{MeV}$ Calibration of JET neutron detectors - Phase 1: calibration and characterization of the neutron source, submitted to Nuclear Fusion, (2017).

[9] M. Pillon, et al., "Characterization of a diamond detector to be used as neutron yield monitor during the invessel calibration of JET neutron detectors in preparation of the DT experiment," Fus. Eng. Des., 106, pp. 9398 (2016).

[10] P. Sauvan, et al., "New capabilities for Monte
Carlo simulation of deuteron transport and secondary product generation," NIMA, 614, pp. 323-330 (2010).

[11] A. Čufar, et al., "Calculations support JET neutron yield calibration: Modelling of neutron emission from a compact DT neutron generator," NIMA, 847, pp. 199-204 (2017). 